\title{
CD44 expression in stem cells and niche microglia/macrophages following ischemic stroke
}

\author{
Rikako Sawada $^{1,2}$, Akiko Nakano-Doi ${ }^{1,3}$, Tomohiro Matsuyama ${ }^{3}$, Nami Nakagomi ${ }^{4}$, Takayuki Nakagomi ${ }^{1,3}$ \\ ${ }^{1}$ Institute for Advanced Medical Sciences, Hyogo College of Medicine, Nishinomiya, Hyogo, Japan; ${ }^{2}$ Graduate School of Science and Technology, \\ Kwansei Gakuin University, Sanda, Hyogo, Japan; ${ }^{3}$ Department of Therapeutic Progress in Brain Diseases, ${ }^{4}$ Department of Surgical Pathology, \\ Hyogo College of Medicine, Nishinomiya, Hyogo, Japan \\ Contributions: (I) Conception and design: R Sawada, T Matsuyama, T Nakagomi; (II) Administrative support: T Nakagomi; (III) Provision of study \\ materials or patients: None; (IV) Collection and assembly of data: R Sawada, A Nakano-Doi; (V) Data analysis and interpretation: R Sawada, A \\ Nakano-Doi, T Matsuyama, N Nakagomi, T Nakagomi; (VI) Manuscript writing: All authors; (VII) Final approval of manuscript: All authors. \\ Correspondence to: Takayuki Nakagomi, MD, PhD. Institute for Advanced Medical Sciences, Hyogo College of Medicine, 1-1 Mukogawacho, \\ Nishinomiya, Hyogo, 663-8501, Japan. Email: nakagomi@hyo-med.ac.jp.
}

\begin{abstract}
Background: CD44, an adhesion molecule in the hyaluronate receptor family, plays diverse and important roles in multiple cell types and organs. Increasing evidence is mounting for CD44 expression in various types of stem cells and niche cells surrounding stem cells. However, the precise phenotypes of CD $44^{+}$cells in the brain under pathologic conditions, such as after ischemic stroke, remain unclear.

Methods: In the present study, using a mouse model for cerebral infarction by middle cerebral artery (MCA) occlusion, we examined the localization and traits of $\mathrm{CD} 44^{+}$cells.

Results: In sham-mice operations, CD44 was rarely observed in the cortex of MCA regions. Following ischemic stroke, $\mathrm{CD}_{4} 4^{+}$cells emerged in ischemic areas of the MCA cortex during the acute phase. Although CD44 at ischemic areas was, in part, expressed in stem cells, it was also expressed in hematopoietic lineages, including activated microglia/macrophages, surrounding the stem cells. CD44 expression in microglia/ macrophages persisted through the chronic phase following ischemic stroke.

Conclusions: These data demonstrate that CD44 is expressed in stem cells and cells in the niches surrounding them, including inflammatory cells, suggesting that CD44 may play an important role in reparative processes within ischemic areas under neuroinflammatory conditions; in particular, strokes.
\end{abstract}

Keywords: CD44; ischemic stroke; microglia; neuroinflammation; stem cell niche

Received: 05 February 2020; Accepted: 18 February 2020; Published: 10 March 2020.

doi: $10.21037 /$ sci.2020.02.02

View this article at: http://dx.doi.org/10.21037/sci.2020.02.02

\section{Introduction}

CD44 is an adhesion molecule that plays essential roles in various cells and multiple organs through a number of signaling pathways (1). Although the precise function of CD44 in the central nervous system (CNS) remains unclear, previous studies demonstrate that $\mathrm{CD} 44$ plays an essential role in regulating the neural cell functions; such as axon guidance, synaptic transmission, oligodendrocyte and astrocyte differentiation, and brain protection and regeneration after injury $(2,3)$. In the $\mathrm{CNS}$, it is well- documented that CD44 is expressed in various neural cell types, such as neurons (4), astrocytes (4-6), and oligodendrocytes $(6,7)$. However, increasing evidence shows that CD44 is observable in neural stem/progenitor cells (NSPCs) $(8,9)$. NSPCs reside in a perivascular niche (10) and produce all neural cell types, including neurons, astrocytes, and oligodendrocytes (11). In addition, Naruse and colleagues found that CD44 expression is dynamically altered from NSPCs to neurons and astrocytes in the developing brain (4), indicating that CD44 is first expressed in NSPCs rather than mature neural cells. 
In various organs outside the CNS, CD44 is expressed in multipotent stem cells, such as mesenchymal stem cells (MSCs) $(12,13)$ and adipose-derived stem cells (ADSCs) $(14,15)$, which localize at the perivascular niche of bone marrows and fat pads, respectively. Although the precious traits of multipotent vascular stem cells in the CNS remain unclear, increasing evidence indicates that brain pericytes are likely multipotent vascular stem cells (16) because of their perivascular localization and multipotency (17-19). In support of this viewpoint, we previously demonstrated that brain pericytes/perivascular cells following ischemia exhibit multipotency (20); thus, ischemia-induced stem cells (iSCs) have the potential to differentiate into neural and also mesoderm lineages (20-29), consistent with the traits of multipotent pericytes (17,30-34). Further, we recently demonstrated that iSCs express CD44 $(21,35,36)$ and that iSCs can differentiate into various neural cell types, including microglia/macrophages lineages (22). As microglia/macrophages function in stem cell niches within the brain $(37,38)$, this suggests that CD44 can be expressed stem cells and the niche cells surrounding them following ischemic strokes.

However, the precise traits of $\mathrm{CD} 44^{+}$cells in brain pathology brains remain unclear. In the present study, using a mouse model of cerebral infarction, we investigated the localization and phenotypes of $\mathrm{CD} 44^{+}$cells in the brain.

\section{Methods}

\section{Induction of focal cerebral ischemia}

All experimental procedures were approved by the Animal Care Committee of the Hyogo College of Medicine (License number: 17-051, 18-061). We used adult 6-10 week-old male mice (CB-17/Icr-+/+Jcl mice (CB-17 mice; Clea Japan Inc., Tokyo, Japan); B6.Cg-Tg(Nes-EGFP)1Yamm transgenic mice (Nestin-GFP mice) (39). Nestin-GFP mice were provided by the RIKEN BRC (Ibaraki, Japan) through the National Bio-Resource Project of the MEXT/AMED, Japan. Permanent focal cerebral ischemia was produced in the mice by ligation and interruption of the distal portion of the left middle cerebral artery (MCA) $(22,24-26,40)$.

\section{Preparation of samples from brains}

CB-17 mice were anesthetized with sodium pentobarbital and transcardially perfused with $4 \%$ paraformaldehyde (PFA) as described previously $(20,35,41)$. Next, whole brains were carefully removed, subjected to post-fixation with 4\% PFA, and processed for paraffin embedding. Samples were then cut into $8-\mu \mathrm{m}$ sections, followed by immunohistochemistry. In another experiment, fixed brains obtained from CB-17 mice or Nestin-GFP mice were cryoprotected in $30 \%$ sucrose, frozen at $-80^{\circ} \mathrm{C}$, and cut into $16-\mu \mathrm{m}$ sections using a cryostat for double-staining using immunohistochemistry.

\section{Immunohistochemistry}

Immunohistochemistry was performed as previously described (42). In brief, after deparaffinization, sections were subjected to heat treatment by microwave for epitope retrieval in a citrate buffer solution $(\mathrm{pH}$ 6.0) (Dako, Glostrup, Denmark) for $10 \mathrm{~min}$. Then, samples were incubated with a primary antibody against CD44 (1:200; rat, Thermo Fisher Scientific, Waltham, MA, USA), followed by reaction with a secondary antibody harboring a universal immunoperoxidase (N-Histofine Simple Stain Mouse MAX PO, Nichirei Corporation, Japan). Sections were stained by 3,3'-diaminobenzide tetrahydrochloride (DAB; Invitrogen, California, USA) and counterstained with hematoxylin. Images were captured using a microscope (Olympus, Tokyo, Japan).

In separate experiments, brain sections (16- $\mu \mathrm{m}$ thick) were subjected to double-stained immunohistochemistry as previously described (23-26,40-43). In brief, sections were stained using primary antibodies against CD44 (1:200; rat, Thermo Fisher Scientific, 1:200; sheep, R\&D systems, Minneapolis, MN, USA), microtubule-associated protein 2 (MAP2) (1:500; mouse, Sigma-Aldrich, St. Louis, MO, USA), MBP (1:100; mouse, R\&D systems), glial fibrillary acidic protein (GFAP) (1:500; mouse, Millipore, Temecula, CA, USA), GFP (1:1,000; rabbit, Abcam), CD31 (1:100, rat, BD Pharmingen, San Diego, CA, USA), PDGFR $\beta$ (1:500, goat, R\&D systems), CD45 (1:200; rat, R\&D systems), Iba1 (1:500; rabbit, Wako, Osaka, Japan), CD86 (1:100; rat, Thermo Fisher Scientific), and CD206 (1:50; goat, R\&D systems). After washing in PBS, sections were stained using Alexa Fluor 488- or 555-conjugated secondary antibodies (1:500; Molecular Probes, Eugene, OR, USA). Cell nuclei were stained with 4',6-diamidino-2-phenylindole (1:500; DAPI, Kirkegaard \& Perry Laboratories, Inc., Gaithersburg, MD, USA). Images were captured using a confocal laser microscope (LSM780; Carl Zeiss AG, Oberkochen, Germany). 


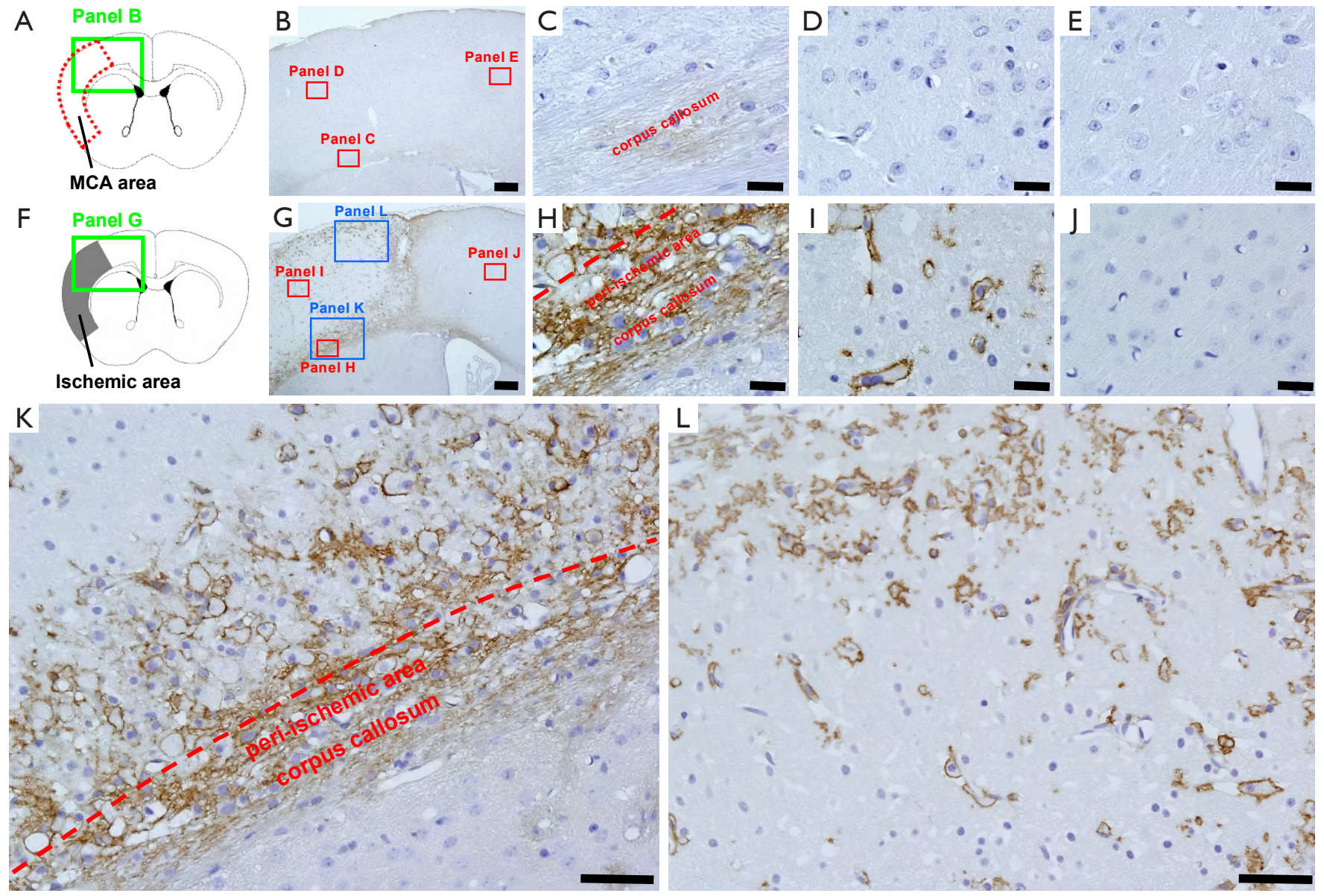

Figure $1 \mathrm{CD}^{4} 4^{+}$cells emerge within ischemic areas during acute phase following stroke. Immunohistochemistry at post-stroke day 7 (A-E) displays weak CD44 staining in the corpus callosum in sham-operated mice (B,C). CD44 was rarely observed in cells of MCA (B and D) and ACA regions (B and E). On day 7 after ischemic stroke (F-I), in addition to the increased expression of CD44 cells in the corpus callosum (G and $\mathrm{H})$, many $\mathrm{CD}_{4} 4^{+}$cells also emerged in peri-ischemic areas $(\mathrm{G}, \mathrm{H}$, and $\mathrm{K})$ and ischemic cores $(\mathrm{G}, \mathrm{I}, \mathrm{K}$, and $\mathrm{L})$. In contrast, CD44 cells were rarely observed in ipsilateral non-ischemic areas $(\mathrm{G}$ and $\mathrm{J})$. Results displayed are representative of three replicates $(\mathrm{N}=3)$. Scale bars $=200 \mu \mathrm{m}$ (B and G), $20 \mu \mathrm{m}$ (C, D, E, H, I, and J), and $50 \mu \mathrm{m}$ (K and L). MCA, middle cerebral artery; ACA, anterior cerebral artery.

\section{Results}

\section{Expression patterns of $\mathrm{CD}_{4} 4^{+}$cells during the acute phase following ischemic stroke}

We first examined the localization of $\mathrm{CD}_{4} 4^{+}$cells following ischemic stroke (Figure 1). In sham-operated mice (Figure $1 A, B, C, D, E$ ), we observed weak staining for $\mathrm{CD} 44^{+}$ cells in the corpus callosum (Figure $1 B, C$ ). However, CD44 ${ }^{+}$ cells were rarely observed in the cells of cortex, which is fed by the MCA (Figure 1B,D) and the anterior cerebral artery (ACA) (Figure 1B,E). However, on day 7 after ischemic stroke (Figure 1F,G,H,I), in addition to enhanced expression of $\mathrm{CD} 44^{+}$cells at the corpus callosum (Figure 1G,H), many
$\mathrm{CD}_{4} 4^{+}$cells appeared in peri-ischemic areas (Figure 1G,H,K) and in ischemic cores (Figure 1G,I,K,L), while they were rarely observed in ipsilateral non-ischemic areas (Figure 1G,7).

\section{CD44 expression by neural lineages including NSPCs following ischemic stroke}

Thus far, our data indicates that $\mathrm{CD} 44^{+}$cells are dramatically increased within ischemic areas following stroke. Previous studies demonstrate that CD44 is expressed in various types of neural cells, including neurons (4), oligodendrocytes (6,7), and astrocytes (4-6). Thus, using brain sections at post-stroke day 7 , we examined 
A

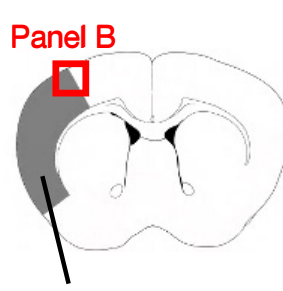

Ischemic area

E

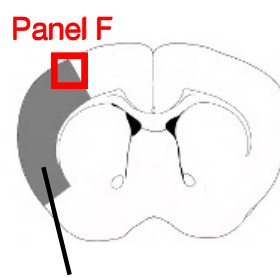

Ischemic area

I

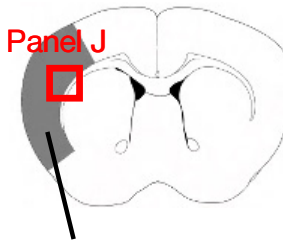

Ischemic area

M

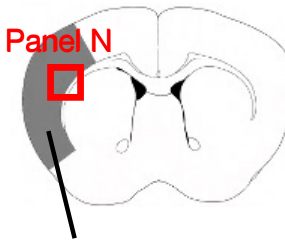

Ischemic area
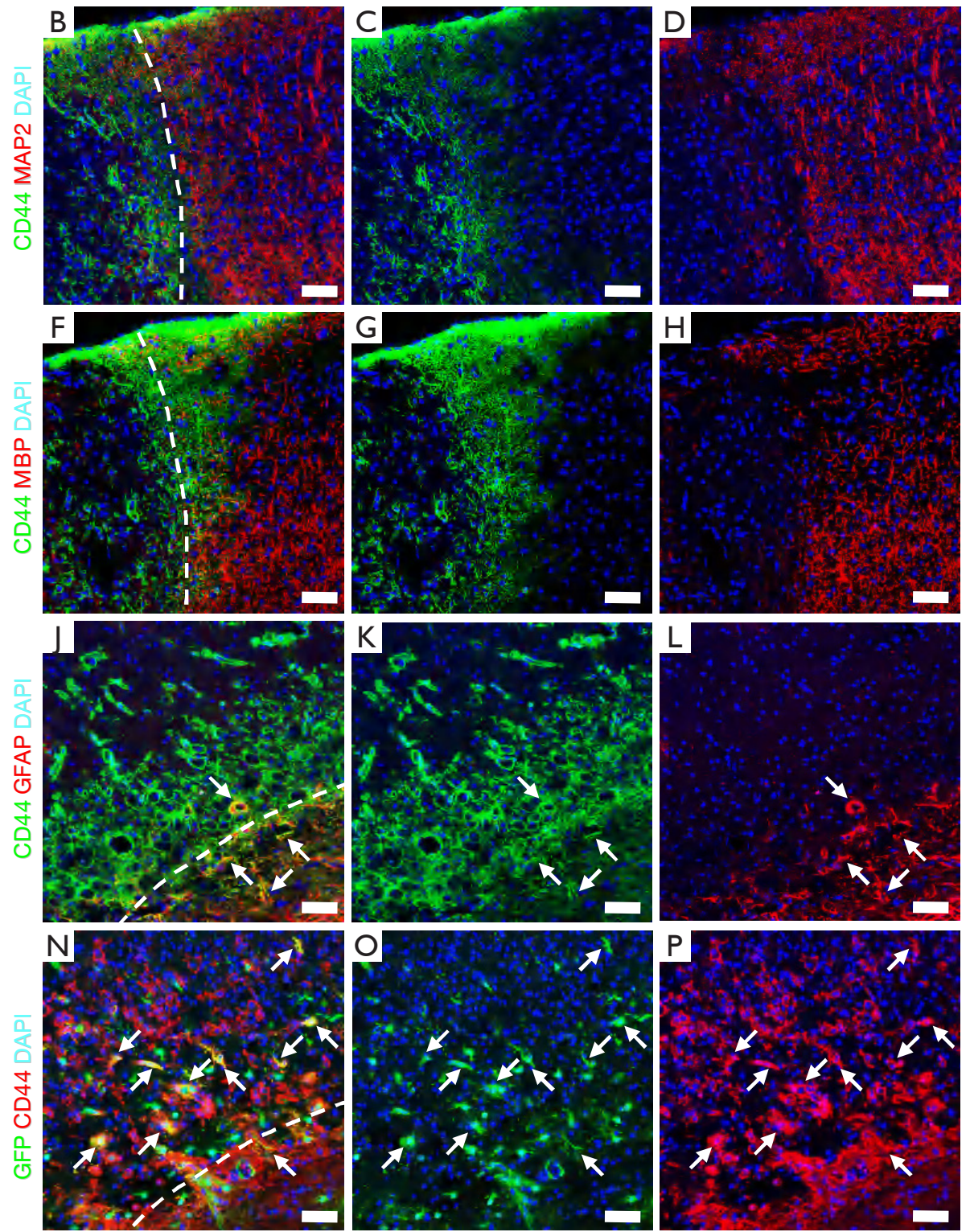

Figure $2 \mathrm{CD}_{4} 4^{+}$cells within ischemic areas are observed in neural lineage cells, including NSPCs. Immunohistochemistry at post-stroke day 7 (A-H) displays CD44+ cells within ischemic areas do not express MAP2 [CD44 (B, C: green), MAP2 (B, D: red), and DAPI (B-D: blue)] or MBP [CD44 (F, G: green), MBP (F, H: red), and DAPI (F-H: blue)]. Immunohistochemistry at post-stroke day 7 (I-L) demonstrates that CD44 is expressed in GFAP' astrocytes primarily localized in peri-ischemic areas [CD44 (J, K: green), GFAP (J, L: red), and DAPI (JL: blue)] (arrows). Immunohistochemistry at post-stroke day 7 (M-P) display CD44 expression in GFP ${ }^{+}$NSPCs which localize in ischemic cores and peri-ischemic areas [GFP (N, O: green), CD44 (N, P: red), and DAPI (N-P: blue)] (arrows). Results displayed are representative of three replicates $(\mathrm{N}=3)$. Scale bars $=50 \mu \mathrm{m}(\mathrm{B}, \mathrm{C}, \mathrm{D}, \mathrm{F}, \mathrm{G}, \mathrm{H}, \mathrm{J}, \mathrm{K}, \mathrm{L}, \mathrm{N}, \mathrm{O}$, and $\mathrm{P})$.

whether CD44 is expressed in our cerebral infarctions. Immunohistochemistry showed that $\mathrm{CD} 44^{+}$cells within ischemic areas did not express the neuronal marker MAP2 (Figure $2 A, B, C, D$ ) or then oligodendrocytic marker MBP (Figure $2 E, F, G, H$ ). In addition, $\mathrm{CD} 44^{+}$cells in peri-ischemic areas including the corpus callosum did not express MAP2 or MBP (data not shown). However, some $\mathrm{CD}_{4} 4^{+}$cells in peri-ischemic areas expressed the astrocytic marker GFAP (Figure 2I, $7, K, L$ ).

NSPCs produce various types of neural cells, including 


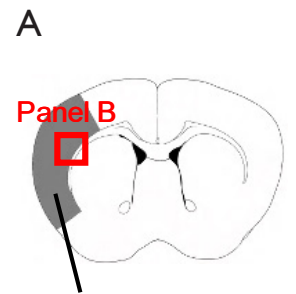

Ischemic area

$\mathrm{F}$

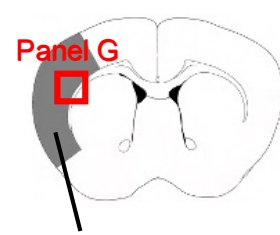

Ischemic area

$\mathrm{K}$

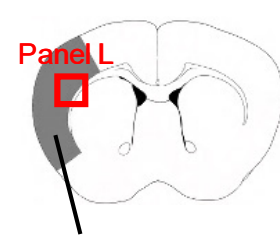

Ischemic area
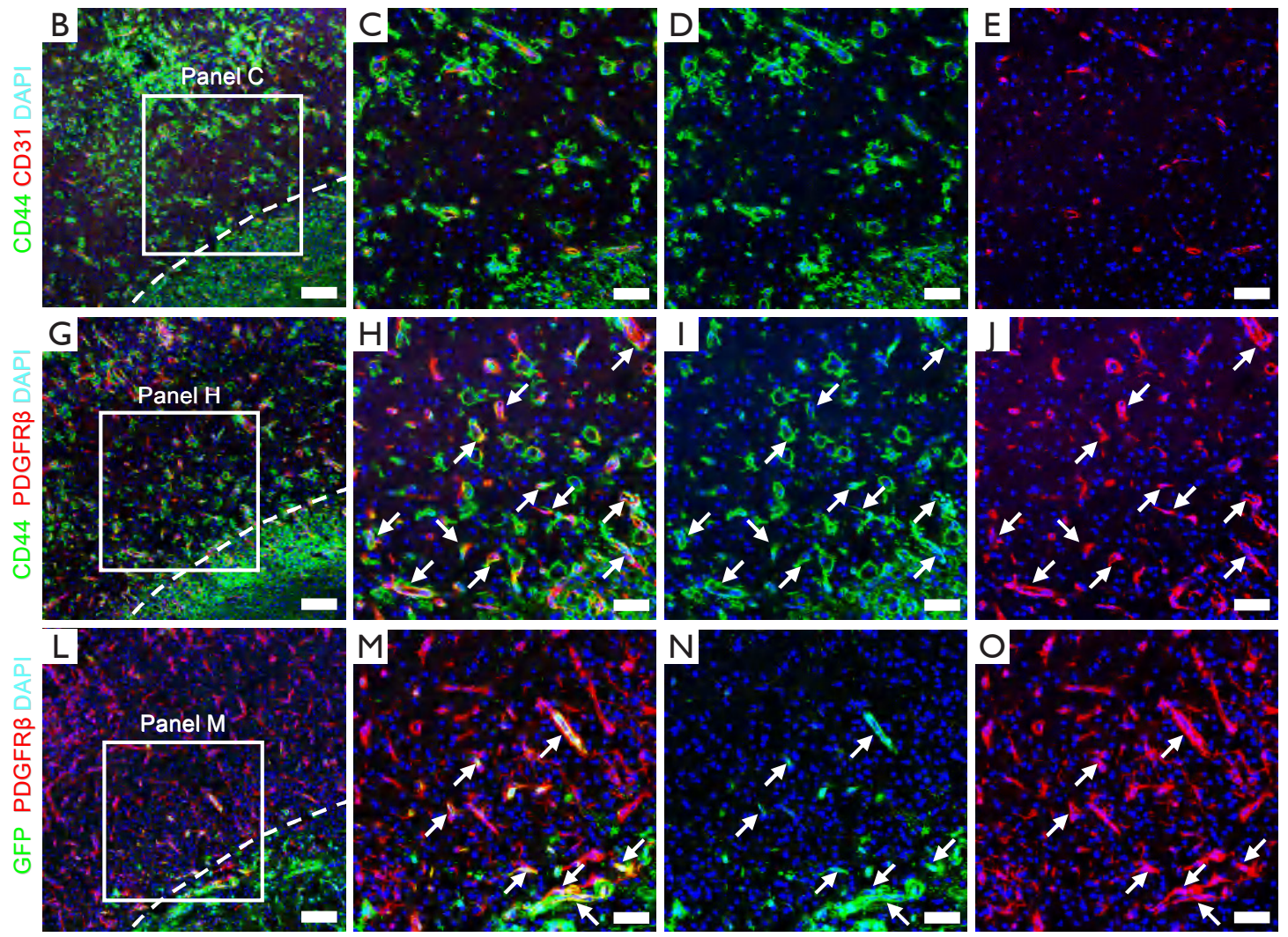

Figure 3 Ischemia induces CD44 expression within some stem cells in stroke-affected areas. Immunohistochemistry at post-stroke day 7 (A-O) demonstrates that CD44 cells do not express CD31 [CD44 (B, C, and D: green), CD31 (B, C, and E: red), and DAPI (B-E: blue)] but do express PDGFR $\beta$ [CD44 (G, H, and I: green), PDGFR $\beta$ (G, H, and J: red), and DAPI (G-J: blue)] (arrows). Immunohistochemistry at post-stroke day 7 (K-O) displays that some GFP ${ }^{+}$cells co-express PDGFR $\beta$ [GFP (L M, and N: green), PDGFR $(\mathrm{L}, \mathrm{M}$, and O: red), and DAPI (L-O: blue)] (arrows). Results displayed are representative of three replicates $(\mathrm{N}=3)$. Scale bars $=100 \mu \mathrm{m}(\mathrm{B}, \mathrm{G}$, and L) and $50 \mu \mathrm{m}(\mathrm{C}, \mathrm{D}$, $\mathrm{E}, \mathrm{H}, \mathrm{I}, \mathrm{J}, \mathrm{M}, \mathrm{N}$, and $\mathrm{O})$.

neurons, astrocytes, and oligodendrocytes (11). In addition, previous studies showed that CD44 is expressed in NSPCs $(8,9)$. Furthermore, we previously demonstrated that NSPCs develop in ischemic cores and in peri-ischemic areas following stroke $(26,40)$. Thus, using nestin-GFP transgenic mice, we investigated whether $\mathrm{GFP}^{+}$cells at the site of ischemic areas express CD44. Using immunohistochemistry, we observed that GFP, under the control of the nestin (a NSPC marker) promoter, was present in ischemic cores and peri-ischemic areas and that some $\mathrm{GFP}^{+}$cells co-expressed CD44 (Figure 2M,N,O,P).

\section{CD44 expression by ischemia-induced stem cells}

The above findings demonstrate that CD44 is expressed in neural lineages, including NSPCs, after ischemic stroke.
However, evidence exists for CD44 expression in various types of stem cells, including MSCs $(12,13)$ and ADSCs $(14,15)$, as well as NSPCs $(8,9)$. Although the precise origin of MSCs and ADSCs is unclear, increasing evidence indicates that they have similar traits to those of pericytes (44-48). In addition, we demonstrated that brain pericytes following ischemia develop into iSCs $(20,24,43)$ and that CD44 is expressed in iSCs that co-express nestin as well as PDGFR $\beta(21,35,36)$. Thus, we investigated whether CD44 is expressed in iSCs. Immunohistochemistry identified CD $44^{+}$cells at the site of ischemic areas localizing around $\mathrm{CD} 31^{+}$endothelial cells (Figure $3 A, B, C, D, E$ ) and that they also expressed PDGFR $\beta$ (Figure $3 F, G, H, I, 7$ ). Furthermore, some $\mathrm{GFP}^{+}$cells in ischemic cores and peri-ischemic areas co-expressed PDGFR $\beta$ (Figure $3 K, L, M, N, O$ ). These results indicate that $\mathrm{CD} 44$ is, in part, expressed in iSCs; which 


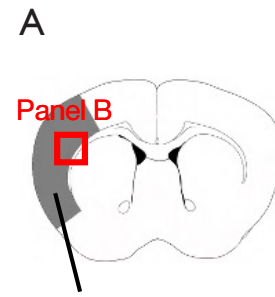

Ischemic area

$\mathrm{F}$

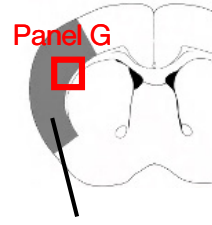

Ischemic area

K

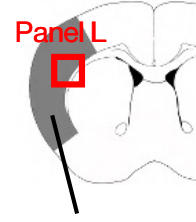

Ischemic area

$P$

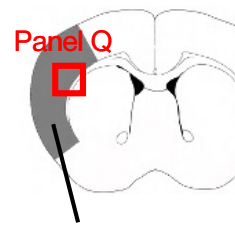

Ischemic area
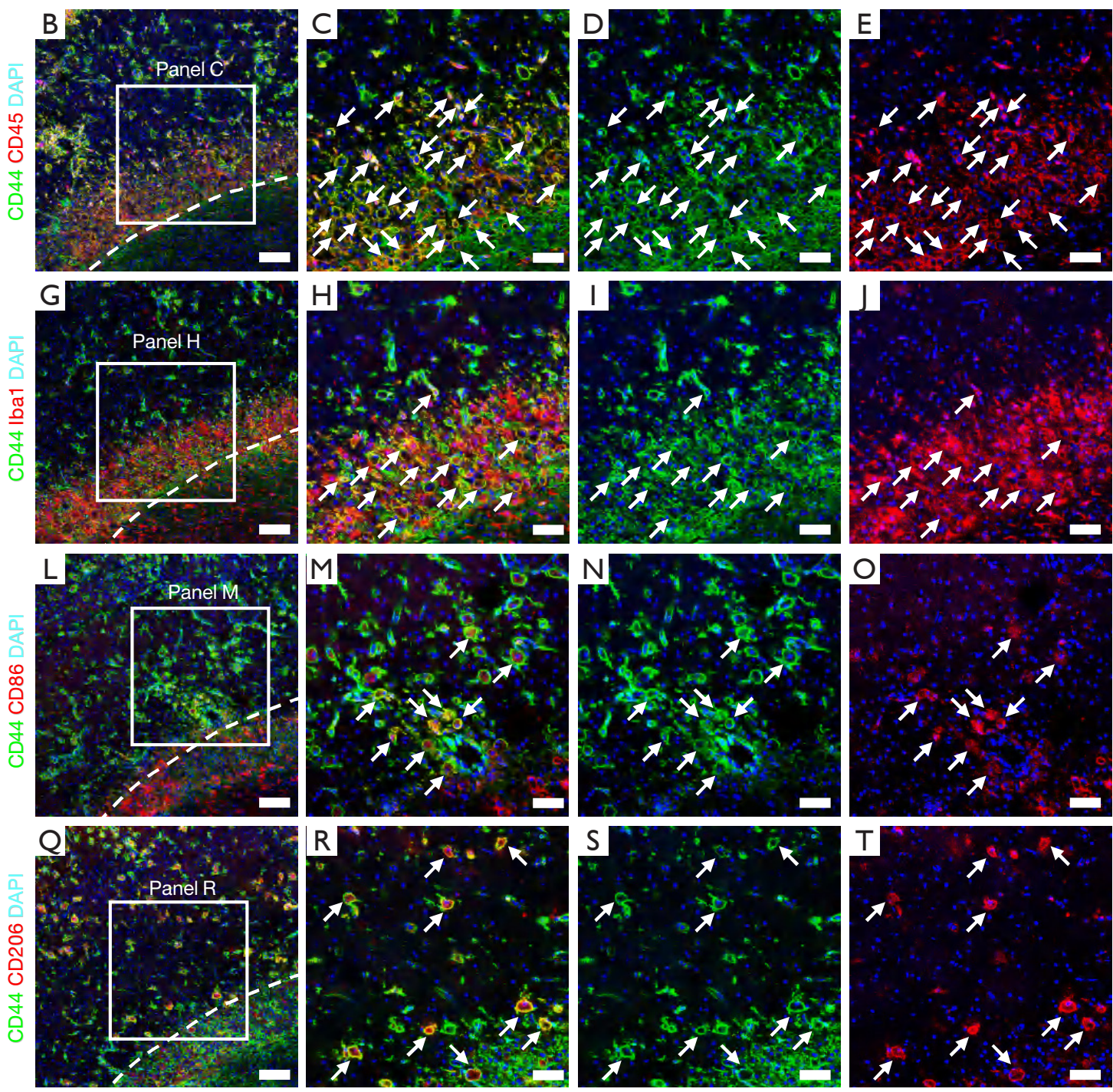

Figure 4 CD44 is expressed in hematopoietic cells including microglia/macrophages within ischemic areas. Immunohistochemistry at poststroke day 7 (A-L) demonstrates that some CD44+ cells within ischemic areas express CD45 [CD44 (B, C, and D: green), CD45 (B, C, and E: red), and DAPI (B-E: blue)] (arrows) and other various microglial/macrophage markers, including Iba1 [CD44 (G, H, and I: green), Iba1 (G, H, and J: red), and DAPI (G-J: blue)] (arrows), CD86 [CD44 (L, M, and N: green), CD86 (L, M, and O: red), and DAPI (L-O: blue)] (arrows), and CD206 [CD44 (Q, R, and S: green), CD206 (Q, R, and T: red), and DAPI (Q-T: blue)] (arrows). Results displayed are representative of three replicates $(\mathrm{N}=3)$. Scale bars $=100 \mu \mathrm{m}(\mathrm{B}, \mathrm{G}, \mathrm{L}$, and Q) and $50 \mu \mathrm{m}(\mathrm{C}, \mathrm{D}, \mathrm{E}, \mathrm{H}, \mathrm{I}, \mathrm{J}, \mathrm{M}, \mathrm{N}, \mathrm{O}, \mathrm{R}, \mathrm{S}$, and T).

likely originate from brain pericytes.

\section{CD44 expression by bematopoietic lineages including microglia/macrophages}

Given the variety of CD44 expression we observe in our infarctions, we investigated whether CD44 is expressed in stem cell niches (e.g., hematopoietic cells including inflammatory cells) surrounding NSPCs and/or iSCs. Immunohistochemistry at post-stroke day 7 demonstrated that many $\mathrm{CD} 44^{+}$cells, in particularly those with round shapes, expressed the hematopoietic marker CD45 (Figure $4 A, B, C, D, E)$. As CD45 is known to be expressed in microglial lineages during brain pathologies $(5,49)$, we further examined whether CD44 is expressed in microglia/ marophages following ischemia. Immunohistochemistry 
A

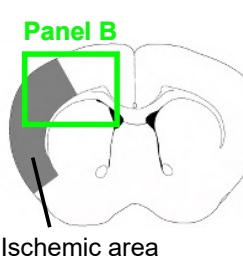

$\mathrm{F}$
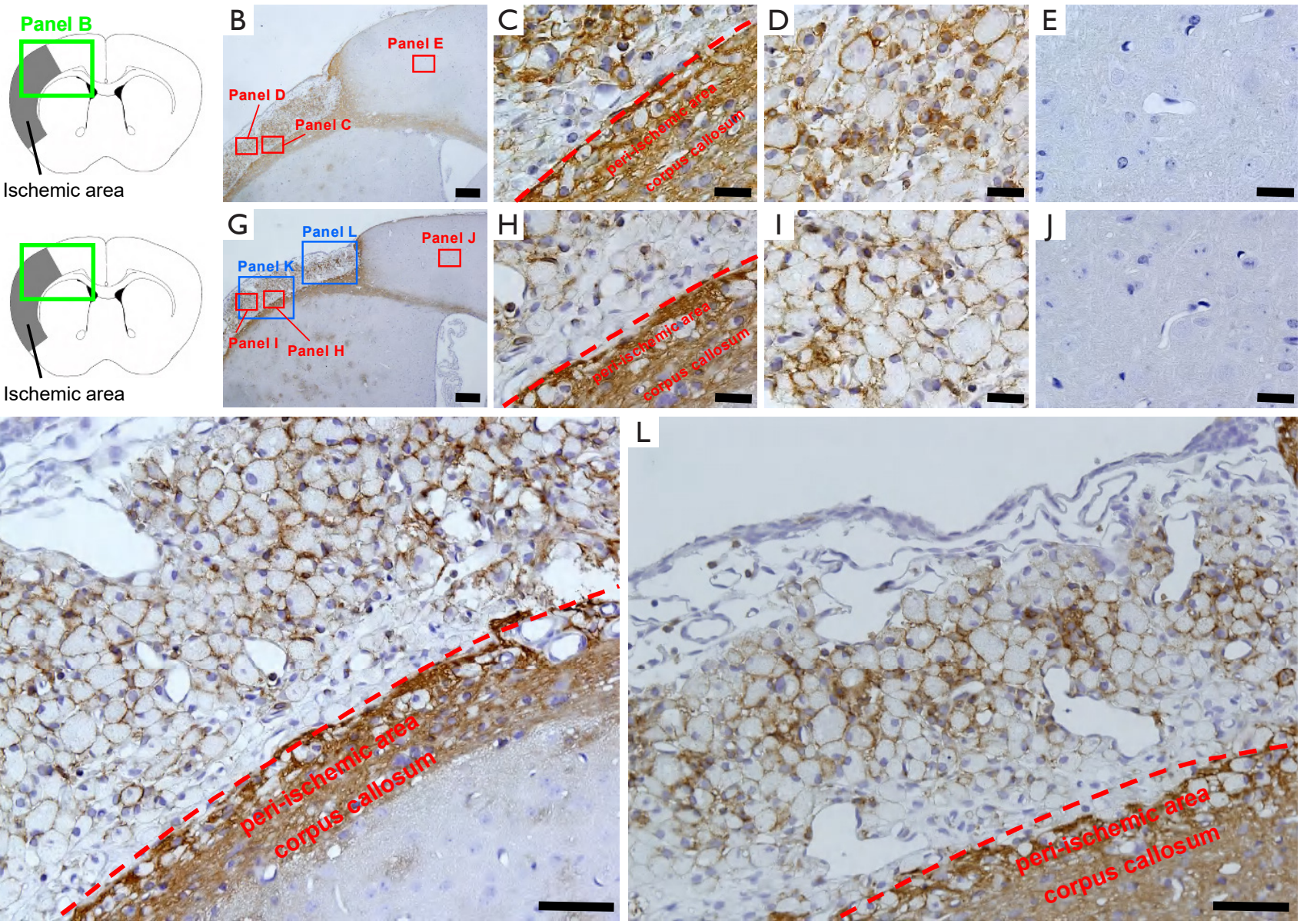

Figure 5 CD44 expression by microglia/macrophages within ischemic areas persists through chronic phases. Immunohistochemistry at poststroke 2 (A-E) and 4 weeks (F-L) demonstrates that CD44 expression by microglia/macrophages within ischemic areas lasts during subacute (B-E) and chronic periods post-stroke (G-L). Results displayed are representative of three replicates $(\mathrm{N}=3)$. Scale bars $=200 \mu \mathrm{m}(\mathrm{B}, \mathrm{G})$, $20 \mu \mathrm{m}(\mathrm{C}, \mathrm{D}, \mathrm{E}, \mathrm{H}, \mathrm{I}, \mathrm{J})$, and $50 \mu \mathrm{m}(\mathrm{K}, \mathrm{L})$.

at post-stroke day 7 indicated that some $\mathrm{CD} 44^{+}$cells in ischemic cores and peri-ischemic areas expressed the microglia/macrophage cell marker Iba1 (Figure 4F,G,H,I,7). These results indicate that, under ischemic stroke, CD44 is expressed in stem cell niches including activated microglia/ macrophages as well as stem cell populations.

As activated microglia/macrophages are known to polarize into two distinct subtypes (pro-inflammatory M1 and anti-inflammatory M2 types) (50-52), we next investigated whether $\mathrm{CD} 44^{+}$cells in ischemic areas expressing the M1 marker CD86 and/or M2 marker CD206. Immunohistochemistry demonstrated that some CD $44^{+}$ cells at these areas co-expressed CD86 (Figure $4 K, L, M, N, O$ ) and CD206 (Figure 4P,Q,R,S,T).

\section{Long-lasting expression of CD44 by microglial macrophages within and around peri-ischemic areas}

Our final experiments in this study investigated the fate of $\mathrm{CD}_{4} 4^{+}$cells at later time points following ischemia. Immunohistochemistry was performed at 2 (Figure $5 A, B, C, D, E)$ and 4 weeks after ischemic stroke (Figure $5 F, G, H, I, \mathcal{F}, K, L)$. Immunohistochemistry displayed $\mathrm{CD} 44^{+}$cells in the corpus callosum (2 weeks after ischemic stroke, Figure 5C; 4 weeks after ischemic stroke, Figure $5 H, K, L)$. In addition, the majority of the ischemic cores and the peri-ischemic areas contained $\mathrm{CD} 44^{+}$foam cells which were morphologically microglia/macrophages (2 weeks after ischemic stroke, Figure 5C,D; 4 weeks after ischemic stroke, Figure $5 H, I, K, L)$. These findings indicate 
that CD44 in ischemic areas is primarily expressed in microglia/macrophages at later time points, including both subacute and chronic phases. These findings also demonstrate that CD44 expression by microglia/ macrophages at these areas is persistent, indicating that CD44 can be used as a marker of activated microglia/ macrophages following brain injuries, such as ischemic stroke.

\section{Discussion}

This study demonstrates that CD44 is expressed in not only stem cells but also stem cell niches such as microglia/ macrophages following stroke. We found that CD44 is expressed in neural lineages, including astrocytes as well as NSPCs, in ischemic areas. Although the precise origin of $\mathrm{CD}_{4} 4^{+}$astrocytes remains unclear, we previously demonstrated that NSCPs isolated from ischemic areas differentiate into various neural cells, including glial cells (40). Thus, it is possible that CD $44^{+} \mathrm{NSPCs}$ are origin of CD $44^{+}$ glial cells. However, the precise relationships between $\mathrm{CD}_{4} 4^{+}$NSPCs and $\mathrm{CD} 44^{+}$glia need clarification; this will be a focus of our future studies.

Under neuroinflammatory conditions, such as after ischemic stroke, it is well-documented that microglia/ macrophages accumulate in the brain (50,52). It has also been reported that brain microglia/macrophages originate from progenitors in the embryonic yolk sac and that they reside in the brain until adulthood under normal conditions (53). Although the precise origin of $\mathrm{CD} 44^{+}$microglia/macrophages under pathological conditions remains unclear, our previous studies $(22)$ and those of others $(54,55)$ demonstrate that activated microglia are, in part, derived from brain pericytes. However, contrary to these finding, it has also been reported that a subset of pericytes originate from hematopoietic lineages, including microglia $(56,57)$. In this study, we found CD44 in ischemic areas was expressed in some $\mathrm{CD} 45^{+}$hematopoietic lineages. As CD44 is also expressed in hematopoietic cells during early CNS development (58), some $\mathrm{CD}_{4} 4^{+}$cells observed in ischemic areas may have the features of immature hematopoietic cells. However, the precise relationship between $\mathrm{CD} 44^{+} / \mathrm{CD} 45^{-}$pericytes and $\mathrm{CD} 44^{+} / \mathrm{CD} 45^{+}$hematopoietic lineage cells in ischemic areas needs to be clarified in further studies.

In this study, we found that many $\mathrm{CD} 44^{+}$cells emerge in ischemic areas, while $\mathrm{CD} 44^{+}$cells are rarely observed in the MCA cells of sham-operated mice. The precise mechanism by which CD44 is selectively enhanced in cells at these areas remains unclear. However, previous studies demonstrate that CD44 expression is up-regulated in microglia/ macrophages after brain injuries, including ischemic stroke $(3,59,60)$, and that CD44 expression is regulated by various cytokines and/or molecules $(61,62)$. This suggests that CD44 can be induced in cells stimulated by several mechanisms, including hypoxia and cytokine production.

Although the precise role of CD44 under ischemic conditions remains unclear, a previous study indicates that CD44-deficiencient mice have lower levels of proinflammatory cytokines after ischemic stroke, resulting in a reduction of ischemic areas, and improved neurological deficits when compared with wild-type control mice (3). These findings suggest that CD44 may function as a negative regulator during ischemic stroke; presumably through an increase of pro-inflammatory cytokines. In contrast to these findings, CD44 deficient mice displayed increased inflammatory responses during experimental autoimmune encephalomyelitis (EAE) when compared to wild-type control mice (63), suggesting that CD44 exerts anti-inflammatory effects. We do not understand the discrepancy of why CD44 displays diverse roles in CD44deficient mice under neuroinflammatory conditions (e.g., ischemic stroke and EAE). However, our current study indicates that CD44 is expressed in activated microglia/ macrophages with two different phenotypes, known as proinflammatory M1 and anti-inflammatory M2 types. Thus, the different results obtained in previous studies regarding CD44-deficient mice may be attributable to the diverse effects of CD44 in microglia/macrophages.

In addition to CD44, the ligands of CD44, such as hyaluronic acid (HA) (64) and osteopontin (OPN) (65), play important roles in the reparative process after injury. Although the precise function of CD44 as a receptor for HA remains unclear, multiple reports demonstrate that implantation of HA scaffolds has various positive effects on CNS regeneration, in part through inhibition of glial scar formation (66-68). OPN is a glycoprotein that was originally purified from bone. However, OPN is expressed in various types of cells, including macrophages $(69,70)$. Thus, it is possible that $\mathrm{CD} 44^{+}$microglia/macrophages also secrete OPN following ischemic stroke. Furthermore, it has been reported that CD44-OPN signaling promotes the proliferation of CNS stem cells, including NSPCs $(71,72)$, neuronal differentiation (72), and axon growth (73). Although the precious roles of microglia/macrophages in during brain pathologies are still unclear, microglia/ macrophages are known to have important roles in stem cell 
niches $(37,38)$. Thus, $\mathrm{CD}_{4} 4^{+}$microglia/macrophages may alter the fates of $\mathrm{CD} 44^{+}$stem cells, such as NSPCs and/ or iSCs, following ischemic stroke; thereby regulating the neural reparative processes during brain injuries. However, the precise relationship between $\mathrm{CD} 44^{+}$stem cells and $\mathrm{CD} 44^{+}$microglia/macrophages in stem cell niches needs to be elucidated by future studies.

In conclusion, we demonstrated that CD44 is expressed, not only stem cells, but also in niche microglia/macrophages following ischemic stroke, suggesting that CD44 plays important roles during reparative processes under neuroinflammatory conditions. However, the precise traits and roles of $\mathrm{CD}_{4} 4^{+}$cells in during brain pathologies require further studies.

\section{Acknowledgments}

We would like to thank A. Narita for technical assistance. Funding: This work was partially supported by Grant-inAid for researchers, Hyogo College of Medicine (2018; to A Nakano-Doi).

\section{Footnote}

Conflicts of Interest: Takayuki Nakagomi serves as the unpaid Editorial Board Member of Stem Cell Investigation. Department of Therapeutic Progress in Brain Diseases is financially supported by Daiichi Sankyo Co., Ltd., Nippon Zoki Pharmaceutical Co., Ltd., and CLEA Japan, Inc. The sponsors had no roles in this study, including those of study design, data collection, data analysis, data interpretation, and manuscript writing. The other authors have no conflicts of interest to declare.

Ethical Statement: The authors are accountable for all aspects of the work in ensuring that questions related to the accuracy or integrity of any part of the work are appropriately investigated and resolved. All experimental procedures were approved by the Animal Care Committee of the Hyogo College of Medicine (License number: 17-051, 18-061). All procedure performed in this study involving animal experiments were in accordance with the ethical standards as described in the methods sections.

Open Access Statement: This is an Open Access article distributed in accordance with the Creative Commons Attribution-NonCommercial-NoDerivs 4.0 International License (CC BY-NC-ND 4.0), which permits the non- commercial replication and distribution of the article with the strict proviso that no changes or edits are made and the original work is properly cited (including links to both the formal publication through the relevant DOI and the license). See: https://creativecommons.org/licenses/by-nc$\mathrm{nd} / 4.0 /$.

\section{References}

1. Ponta H, Sherman L, Herrlich PA. CD44: from adhesion molecules to signalling regulators. Nat Rev Mol Cell Biol 2003;4:33-45.

2. Dzwonek J, Wilczynski GM. CD44: molecular interactions, signaling and functions in the nervous system. Front Cell Neurosci 2015;9:175.

3. Wang X, Xu L, Wang H, et al. CD44 deficiency in mice protects brain from cerebral ischemia injury. J Neurochem 2002;83:1172-9.

4. Naruse M, Shibasaki K, Yokoyama S, et al. Dynamic changes of CD44 expression from progenitors to subpopulations of astrocytes and neurons in developing cerebellum. PLoS One 2013;8:e53109.

5. Matsumoto T, Imagama S, Hirano K, et al. CD44 expression in astrocytes and microglia is associated with ALS progression in a mouse model. Neurosci Lett 2012;520:115-20.

6. Moretto G, Xu RY, Kim SU. CD44 expression in human astrocytes and oligodendrocytes in culture. J Neuropathol Exp Neurol 1993;52:419-23.

7. Tuohy TM, Wallingford N, Liu Y, et al. CD44 overexpression by oligodendrocytes: a novel mouse model of inflammation-independent demyelination and dysmyelination. Glia 2004;47:335-45.

8. Deboux C, Ladraa S, Cazaubon S, et al. Overexpression of CD44 in neural precursor cells improves trans-endothelial migration and facilitates their invasion of perivascular tissues in vivo. PLoS One 2013;8:e57430.

9. New SE, Alvarez-Gonzalez C, Vagaska B, et al. A matter of identity - Phenotype and differentiation potential of human somatic stem cells. Stem Cell Res 2015;15:1-13.

10. Tavazoie M, Van der Veken L, Silva-Vargas V, et al. A specialized vascular niche for adult neural stem cells. Cell Stem Cell 2008;3:279-88.

11. Reynolds BA, Weiss S. Generation of neurons and astrocytes from isolated cells of the adult mammalian central nervous system. Science 1992;255:1707-10.

12. Crisan M, Yap S, Casteilla L, et al. A perivascular origin for mesenchymal stem cells in multiple human organs. 
Cell Stem Cell 2008;3:301-13.

13. Murray IR, West CC, Hardy WR, et al. Natural history of mesenchymal stem cells, from vessel walls to culture vessels. Cell Mol Life Sci 2014;71:1353-74.

14. Lin G, Garcia M, Ning H, et al. Defining stem and progenitor cells within adipose tissue. Stem Cells Dev 2008; 17:1053-63.

15. Lin CS, Xin ZC, Deng CH, et al. Defining adipose tissue-derived stem cells in tissue and in culture. Histol Histopathol 2010;25:807-15.

16. Lin CS, Lue TF. Defining vascular stem cells. Stem Cells Dev 2013;22:1018-26.

17. Dore-Duffy P, Katychev A, Wang X, et al. CNS microvascular pericytes exhibit multipotential stem cell activity. J Cereb Blood Flow Metab 2006;26:613-24.

18. Karow M, Sanchez R, Schichor C, et al. Reprogramming of pericyte-derived cells of the adult human brain into induced neuronal cells. Cell Stem Cell 2012;11:471-6.

19. Nakagomi T, Nakano-Doi A, Kawamura M, et al. Do Vascular Pericytes Contribute to Neurovasculogenesis in the Central Nervous System as Multipotent Vascular Stem Cells? Stem Cells Dev 2015;24:1730-9.

20. Nakagomi T, Kubo S, Nakano-Doi A, et al. Brain vascular pericytes following ischemia have multipotential stem cell activity to differntiate into neural and vascular lineage cells. Stem Cells 2015;33:1962-74.

21. Tatebayashi K, Tanaka Y, Nakano-Doi A, et al. Identification of Multipotent Stem Cells in Human Brain Tissue Following Stroke. Stem Cells Dev 2017;26:787-97.

22. Sakuma R, Kawahara M, Nakano-Doi A, et al. Brain pericytes serve as microglia-generating multipotent vascular stem cells following ischemic stroke. J Neuroinflammation 2016;13:57.

23. Nakagomi T, Molnar Z, Taguchi A, et al. Leptomeningealderived doublecortin-expressing cells in poststroke brain. Stem Cells Dev 2012;21:2350-4.

24. Nakagomi T, Molnar Z, Nakano-Doi A, et al. Ischemiainduced neural stem/progenitor cells in the pia mater following cortical infarction. Stem Cells Dev 2011;20:2037-51

25. Nakagomi N, Nakagomi T, Kubo S, et al. Endothelial cells support survival, proliferation, and neuronal differentiation of transplanted adult ischemia-induced neural stem/ progenitor cells after cerebral infarction. Stem Cells 2009;27:2185-95.

26. Nakano-Doi A, Nakagomi T, Fujikawa M, et al. Bone marrow mononuclear cells promote proliferation of endogenous neural stem cells through vascular niches after cerebral infarction. Stem Cells 2010;28:1292-302.

27. Nakagomi T, Nakano-Doi A, Narita A, et al. Concise Review: Are Stimulated Somatic Cells Truly Reprogrammed into an ES/iPS-Like Pluripotent State? Better Understanding by Ischemia-Induced Multipotent Stem Cells in a Mouse Model of Cerebral Infarction. Stem Cells Int 2015;2015:630693.

28. Nakagomi T, Nakano-Doi A, Matsuyama T. Leptomeninges: a novel stem cell niche harboring ischemia-induced neural progenitors. Histol Histopathol 2015;30:391-9.

29. Takagi T, Yoshimura S, Sakuma R, et al. Novel Regenerative Therapies Based on Regionally Induced Multipotent Stem Cells in Post-Stroke Brains: Their Origin, Characterization, and Perspective. Transl Stroke Res 2017;8:515-28.

30. Birbrair A, Zhang T, Wang ZM, et al. Role of pericytes in skeletal muscle regeneration and fat accumulation. Stem Cells Dev 2013;22:2298-314.

31. Birbrair A, Zhang T, Wang ZM, et al. Skeletal muscle neural progenitor cells exhibit properties of NG2-glia. Exp Cell Res 2013;319:45-63.

32. Farrington-Rock C, Crofts NJ, Doherty MJ, et al. Chondrogenic and adipogenic potential of microvascular pericytes. Circulation 2004;110:2226-32.

33. Doherty MJ, Ashton BA, Walsh S, et al. Vascular pericytes express osteogenic potential in vitro and in vivo. J Bone Miner Res 1998;13:828-38.

34. Orekhov AN, Bobryshev YV, Chistiakov DA. The complexity of cell composition of the intima of large arteries: focus on pericyte-like cells. Cardiovasc Res 2014;103:438-51.

35. Sakuma R, Takahashi A, Nakano-Doi A, et al. Comparative Characterization of Ischemia-Induced Brain Multipotent Stem Cells with Mesenchymal Stem Cells: Similarities and Differences. Stem Cells Dev 2018;27:1322-38.

36. Beppu M, Nakagomi T, Takagi T, et al. Isolation and Characterization of Cerebellum-Derived Stem Cells in Poststroke Human Brain. Stem Cells Dev 2019;28:528-42.

37. Solano Fonseca R, Mahesula S, Apple DM, et al. Neurogenic Niche Microglia Undergo Positional Remodeling and Progressive Activation Contributing to Age-Associated Reductions in Neurogenesis. Stem Cells Dev 2016;25:542-55.

38. Matsui TK, Mori E. Microglia support neural stem cell maintenance and growth. Biochem Biophys Res Commun 2018;503:1880-4.

39. Yamaguchi M, Saito H, Suzuki M, et al. Visualization 
of neurogenesis in the central nervous system using nestin promoter-GFP transgenic mice. Neuroreport 2000;11:1991-6.

40. Nakagomi T, Taguchi A, Fujimori Y, et al. Isolation and characterization of neural stem/progenitor cells from post-stroke cerebral cortex in mice. Eur J Neurosci 2009;29:1842-52.

41. Nakano-Doi A, Sakuma R, Matsuyama T, et al. Ischemic stroke activates the VE-cadherin promoter and increases VE-cadherin expression in adult mice. Histol Histopathol 2018;33:507-21.

42. Maeda Y, Nakagomi N, Nakano-Doi A, et al. Potential of Adult Endogenous Neural Stem/Progenitor Cells in the Spinal Cord to Contribute to Remyelination in Experimental Autoimmune Encephalomyelitis. Cells 2019. doi: 10.3390/cells8091025.

43. Nakata $M$, Nakagomi T, Maeda $M$, et al. Induction of Perivascular Neural Stem Cells and Possible Contribution to Neurogenesis Following Transient Brain Ischemia/ Reperfusion Injury. Transl Stroke Res 2017;8:131-43.

44. Feng J, Mantesso A, De Bari C, et al. Dual origin of mesenchymal stem cells contributing to organ growth and repair. Proc Natl Acad Sci U S A 2011;108:6503-8.

45. Caplan AI. All MSCs are pericytes? Cell Stem Cell 2008;3:229-30.

46. Corselli M, Chen CW, Crisan M, et al. Perivascular ancestors of adult multipotent stem cells. Arterioscler Thromb Vasc Biol 2010;30:1104-9.

47. Merfeld-Clauss S, Gollahalli N, March KL, et al. Adipose tissue progenitor cells directly interact with endothelial cells to induce vascular network formation. Tissue Eng Part A 2010;16:2953-66.

48. Merfeld-Clauss S, Lupov IP, Lu H, et al. Adipose stromal cells differentiate along a smooth muscle lineage pathway upon endothelial cell contact via induction of activin A. Circ Res 2014;115:800-9.

49. Rustenhoven J, Park TI, Schweder P, et al. Isolation of highly enriched primary human microglia for functional studies. Sci Rep 2016;6:19371.

50. Liu Z, Ran Y, Huang S, et al. Curcumin Protects against Ischemic Stroke by Titrating Microglia/Macrophage Polarization. Front Aging Neurosci 2017;9:233.

51. Liu C, Li Y, Yu J, et al. Targeting the shift from M1 to $\mathrm{M} 2$ macrophages in experimental autoimmune encephalomyelitis mice treated with fasudil. PLoS One 2013;8:e54841.

52. Hu X, Li P, Guo Y, et al. Microglia/macrophage polarization dynamics reveal novel mechanism of injury expansion after focal cerebral ischemia. Stroke 2012;43:3063-70.

53. Alliot F, Godin I, Pessac B. Microglia derive from progenitors, originating from the yolk sac, and which proliferate in the brain. Brain Res Dev Brain Res 1999;117:145-52.

54. Özen I, Deierborg T, Miharada K, et al. Brain pericytes acquire a microglial phenotype after stroke. Acta Neuropathol 2014;128:381-96.

55. Gouveia A, Seegobin M, Kannangara TS, et al. The aPKC-CBP Pathway Regulates Post-stroke Neurovascular Remodeling and Functional Recovery. Stem Cell Reports 2017;9:1735-44.

56. Yamamoto S, Muramatsu M, Azuma E, et al. A subset of cerebrovascular pericytes originates from mature macrophages in the very early phase of vascular development in CNS. Sci Rep 2017;7:3855.

57. Yamazaki T, Nalbandian A, Uchida Y, et al. Tissue Myeloid Progenitors Differentiate into Pericytes through TGF-beta Signaling in Developing Skin Vasculature. Cell Rep 2017;18:2991-3004.

58. Corbel C, Lehmann A, Davison F. Expression of CD44 during early development of the chick embryo. Mech Dev 2000;96:111-4.

59. Wang H, Zhan Y, Xu L, et al. Use of suppression subtractive hybridization for differential gene expression in stroke: discovery of CD44 gene expression and localization in permanent focal stroke in rats. Stroke 2001;32:1020-7.

60. Jones LL, Liu Z, Shen J, et al. Regulation of the cell adhesion molecule CD44 after nerve transection and direct trauma to the mouse brain. J Comp Neurol 2000;426:468-92.

61. Fitzgerald KA, O'Neill LA. Characterization of CD44 induction by IL-1: a critical role for Egr-1. J Immunol 1999;162:4920-7.

62. Foster LC, Arkonac BM, Sibinga NE, et al. Regulation of CD44 gene expression by the proinflammatory cytokine interleukin-1beta in vascular smooth muscle cells. J Biol Chem 1998;273:20341-6.

63. Flynn KM, Michaud M, Madri JA. CD44 deficiency contributes to enhanced experimental autoimmune encephalomyelitis: a role in immune cells and vascular cells of the blood-brain barrier. Am J Pathol 2013;182:1322-36.

64. Litwiniuk M, Krejner A, Speyrer MS, et al. Hyaluronic Acid in Inflammation and Tissue Regeneration. Wounds 2016;28:78-88.

65. Wang $W$, Li P, Li W, et al. Osteopontin activates mesenchymal stem cells to repair skin wound. PLoS One 
2017; 12:e0185346.

66. Tian WM, Hou SP, Ma J, et al. Hyaluronic acid-poly-Dlysine-based three-dimensional hydrogel for traumatic brain injury. Tissue Eng 2005;11:513-25.

67. Hou S, Tian $W, X u Q$, et al. The enhancement of cell adherence and inducement of neurite outgrowth of dorsal root ganglia co-cultured with hyaluronic acid hydrogels modified with Nogo-66 receptor antagonist in vitro. Neuroscience 2006;137:519-29.

68. Lin CM, Lin JW, Chen YC, et al. Hyaluronic acid inhibits the glial scar formation after brain damage with tissue loss in rats. Surg Neurol 2009;72 Suppl 2:S50-4.

69. Murry CE, Giachelli CM, Schwartz SM, et al. Macrophages express osteopontin during repair of myocardial necrosis. Am J Pathol 1994;145:1450-62.

doi: $10.21037 /$ sci.2020.02.02

Cite this article as: Sawada R, Nakano-Doi A, Matsuyama T, Nakagomi N, Nakagomi T. CD44 expression in stem cells and niche microglia/macrophages following ischemic stroke. Stem Cell Investig 2020;7:4.
70. Küry P, Zickler P, Stoll G, et al. Osteopontin, a macrophage-derived matricellular glycoprotein, inhibits axon outgrowth. FASEB J 2005;19:398-400.

71. Pietras A, Katz AM, Ekstrom EJ, et al. Osteopontin-CD44 signaling in the glioma perivascular niche enhances cancer stem cell phenotypes and promotes aggressive tumor growth. Cell Stem Cell 2014;14:357-69.

72. Rabenstein M, Hucklenbroich J, Willuweit A, et al. Osteopontin mediates survival, proliferation and migration of neural stem cells through the chemokine receptor CXCR4. Stem Cell Res Ther 2015;6:99.

73. Ries A, Goldberg JL, Grimpe B. A novel biological function for CD44 in axon growth of retinal ganglion cells identified by a bioinformatics approach. J Neurochem 2007;103:1491-505. 